\title{
Some new integral relation of I- function
}

\author{
Jyoti Mishra ${ }^{1}$ and Vandana ${ }^{2 *}$ \\ ${ }^{1}$ Department of Mathematics, Gyan Ganga Institute of Technology and Sciences, Jabalpur, India \\ ${ }^{2}$ Department of Management Studies, Indian Institute of Technology Madras, Chennai, Tamilnadu, India
}

\begin{abstract}
This paper deals with some new integral relation of I- function of one variable.
\end{abstract}

\section{Introduction}

The I- function of one variable is defined by Saxena [1] and we shall represent here in the following manner:

$$
\begin{aligned}
& I[z]=\quad \mathrm{I}_{p_{i}, q_{i}: r}^{m, n}\left[z \mid \begin{array}{l}
{\left[\left(a_{j}, \alpha_{j}\right)_{1, n}\right],\left[\left(a_{j i}, \alpha_{j i}\right)_{n+1} p_{i}\right]} \\
{\left[\left(b_{j}, \beta_{j}\right)_{1, m}\right],\left[\left(b_{j i}, \beta_{j i}\right)_{m+1}, q_{i}\right]}
\end{array}\right] \\
& =\frac{1}{2 \pi w} \int_{L} \theta(s) z^{s} d s,
\end{aligned}
$$

where $\omega=\sqrt{(-1)}, z(\neq 0)$ is a complex variable and

$$
z^{s}=\exp [s\{\log |z|+w \arg \mathrm{z}\}] \text {. }
$$

In which $\log |z|$ represent the natural logarithm of $|z|$ and $\arg |z|$ is not necessarily the principle value. An empty product is interpreted as unity, also,

$$
\theta(s)=\frac{\prod_{j=1}^{m} \Gamma\left(b_{j}-\beta_{j} s\right) \prod_{j=1}^{n} \Gamma\left(1-a_{j}-\alpha_{j} s\right)}{\sum_{i=1}^{r}\left[\prod_{j=m+1}^{q_{i}} \Gamma\left(1-b_{j i}-\beta_{j i} s\right) \prod_{j=n+1}^{p_{i}} \Gamma\left(a_{j i}-\alpha_{j i} s\right)\right]},
$$

$\mathrm{m}, \mathrm{n}$ and pi $\forall \mathrm{i} \in(1, \ldots . . \mathrm{r})$ are non-negative integers satisfying $0<$ $\mathrm{n}<\mathrm{p}_{\mathrm{i}}, 0<\mathrm{m}<\mathrm{q}_{\mathrm{i}}, \quad \forall \mathrm{i} \in(1, \ldots . \mathrm{r}), \alpha_{\mathrm{ii}},\left(\mathrm{j}=1, \ldots . . \mathrm{p}_{\mathrm{i}} ; \mathrm{i}=1, \ldots \ldots . . \mathrm{r}\right)$ and $\beta_{\mathrm{ji}}\left(\mathrm{j}=1, \ldots . \mathrm{q}_{\mathrm{i}} ; \mathrm{i}=1, \ldots . \mathrm{r}\right)$ are assumed to be positive quantities for standardization purpose. Also $a_{j i}\left(j=1, \ldots, p_{i} ; i=1, \ldots \ldots, r\right)$ and $b_{j i}(j$ $\left.=1, \ldots \ldots ., \mathrm{q}_{\mathrm{i}} ; \mathrm{i}=1, \ldots \ldots, \mathrm{r}\right)$ are complex numbers such that none of the points.

$$
S=\left\{(b n+v)\left|\beta_{h}\right|\right\}, h=1, \ldots \ldots, m ; v=0,1,2, \ldots . .
$$

which are the poles of $\Gamma\left(b_{n}-\beta_{n} S\right), h=1, \ldots \ldots . m$ and the points.

$$
\mathrm{S}=\left\{\left(a_{1}-\eta-1\right)\left|\alpha_{l}\right| l=1, \ldots ., n ; \eta=0,1,2, \ldots .\right.
$$

which are the poles of $\Gamma\left(1-a_{l}+\alpha_{l} s\right)$ coincide with one another, i.e. with

$$
\alpha_{l}\left(b_{n}+v\right) \neq b_{n}\left(a_{l}-\eta-1\right)
$$

for $\mathrm{n}, \mathrm{h}=0,1,2, \ldots . ; \mathrm{h}=1, \ldots ., \mathrm{m} ; \mathrm{l}=1, \ldots ., \mathrm{n}$.

Further, the contour L runs from $-\omega \infty$ to $+\omega \infty$. Such that the poles of $\Gamma\left(b_{n}-\beta_{n} s\right), \mathrm{h}=1 \ldots \ldots, \mathrm{m}$; lie to the right of $\mathrm{L}$ and the poles $\Gamma\left(1-a_{l}+\alpha_{l} s\right), \mathrm{l}=1, \ldots, \mathrm{n}$ lie to the left of $\mathrm{L}$. The integral (1.1) converges, if $|\arg \mathrm{z}|<1 / 2 \mathrm{~B} \pi \quad(\mathrm{B}>0), \mathrm{A}<0$, where

$$
A=\sum_{j=1}^{p_{i}} a_{j i}-\sum_{j=1}^{q_{i}} \beta_{j i} .
$$

and

$$
\begin{aligned}
& B=\sum_{j=1}^{n} \alpha_{j}-\sum_{j=n+1}^{p_{i}} \alpha_{j i}+\sum_{j=1}^{m} \beta_{j}-\sum_{j=m+1}^{q_{i}} \beta_{j i}, \\
& \forall \mathrm{i} \in(1, \ldots \ldots, \mathrm{r}) .
\end{aligned}
$$

Gradshteyin and Ryzhik [2] given table of Integrals, series, Sharma [3] evaluated the integrals involving general class of polynomial with $\mathrm{H}$-function, Srivastava and Garg [4] established some integrals involving a general class of polynomials and the multivariable $\mathrm{H}$ function. Recently, Satyanarayana and Pragathi Kumar [5] has evaluated Some finite integrals involving multivariable polynomials, Agarwal [6] established integral involving the product of Srivastava's polynomials and generalized Mellin-Barnes type of contour integral, Bhattar [7] established some integral formulas involving two $\bar{H}$ - function and multivariable's general class of polynomiyals. Satyanarayana and Pragathi Kumar [5] has evaluated some finite integrals involving multivariable polynomials. Following them, I evaluated some new integrals involving multivariable polynomials, and I-function of one variable.

\section{Formula Required}

The following formulas will be required in our investigation

(i) The second class of multivariable polynomials given by Srivastava $[8,9]$ is defined as follows:

$S_{V_{1}, \ldots, V_{t}}^{U_{1}, \ldots, U_{t}}\left[x_{1}, \ldots, x_{t}\right]=\sum_{K_{1}=0}^{\left[V_{1} / U_{1}\right]} \ldots \sum_{K_{t}=0}^{\left[V_{1} / U_{t}\right]}\left(-V_{1}\right)_{U_{1} k_{1}} \ldots\left(-V_{t}\right)_{U_{t} k_{t}} \mathrm{~A}\left[V_{1}, k_{1} ; \ldots ; V_{t}, k_{t}\right] \frac{x_{1}^{k_{1}}}{k_{1} !} \ldots \frac{x_{t}^{k_{t}}}{k_{t} !}$.

(ii) The first class of multivariable polynomials introduced by Srivastava and Garg [4] is defined as follows:

Correspondence to: Vandana, Department of Management Studies, Indian Institute of Technology Madras, Chennai, Tamil Nadu - 600036, India,, E-mail: vdrai1988@gmail.com

Key words: I- function, multivariable polynomial

Received: October 28, 2016; Accepted: December 24, 2016; Published: December 27, 2016 


$$
S_{V}^{U_{1}, \ldots, U_{t}}\left[x_{1}, \ldots, x_{t}\right]=\sum_{K_{1} \ldots k_{t}=o}^{U_{1} k_{1}+\ldots U_{t} K_{t} \leq V}\left(-V_{1}\right)_{U_{1} k_{1}} \ldots\left(-V_{t}\right)_{U_{t} k_{t}} \mathrm{~A}\left[V_{1}, k_{1} ; \ldots ; V_{t}, k_{t}\right] \frac{x_{1}^{k_{1}}}{k_{1} !} \ldots \frac{x_{t}^{k_{t}}}{k_{t} !}
$$

\section{Some New Finite Integrals Formulae}

In this section we prove two integral formulae, which involving multivariable polynomials, and I function of one variable.

$$
\begin{aligned}
& \int_{-1}^{1}(1-x)^{\rho}(1+x)^{\sigma} \mathrm{S}_{V_{1}, \ldots, V_{t}}^{U_{1}, \ldots, U_{t}}\left[\mathrm{y}_{1}(1-x)^{m_{1}}(1+x)^{n_{1}}, \ldots, \mathrm{y}_{t}(1-x)^{m_{t}}(1+x)^{n_{t}}\right] \\
& \times I_{p_{i}, q_{i}: r}^{m, n}\left[z(1-x)^{g}(1+x)^{h} \mid \begin{array}{l}
{\left[\left(a_{j}, \alpha_{j}\right)_{1, n}\right],\left[\left(a_{j i}, \alpha_{j i}\right)_{n+1, p_{i}}\right]} \\
{\left[\left(b_{j}, \beta_{j}\right)_{1, n}\right],\left[\left(b_{j i}, \beta_{j i}\right)_{n+1, p_{i}}\right]}
\end{array}\right] \mathrm{d} x \\
& =2^{\rho+\sigma+1} \sum_{K_{1}=0}^{\left[V_{1} / U_{1}\right]} \ldots \ldots . \sum_{K_{t}=0}^{\left[V_{,} / U_{t}\right]}\left(-V_{1}\right)_{U_{1} k_{1}} \ldots\left(-V_{t}\right)_{U_{t} K_{t}} \mathrm{~A}\left[\mathrm{~V}_{1}, k_{1} ; \ldots . . \mathrm{V}_{t}, k_{t}\right] \frac{y_{1}^{k_{1}}}{k_{1} !} \ldots \frac{y_{t}^{k_{t}}}{k_{t} !} 2^{\sum_{i=1}^{t}\left(m_{i}+n_{i}\right) k_{i}} \\
& \times I_{p_{i}, q_{i}: r}^{m, n}\left[z 2^{h+g} \mid \begin{array}{l}
\left(-\sigma-\sum_{i=1}^{t} m_{i} k_{i}, h ; 1\right),\left(-\rho-\sum_{i=1}^{t} n_{i} k_{i}, g ; 1\right)\left[\left(a_{j}, \alpha_{j}\right)_{1, n}\right],\left[\left(a_{j i}, \alpha_{j i}\right)_{n+1, p_{i}}\right] \\
{\left[\left(b_{j}, \beta_{j}\right)_{1, n}\right],\left[\left(b_{j i}, \beta_{j i}\right)_{n+1, q_{i}}\right]\left(-\sigma-\rho-\sum_{i=1}^{t}\left(m_{i}+n_{i}\right) k_{i}-1, h+g ; 1\right)}
\end{array}\right]
\end{aligned}
$$

where $m_{i}>0(i=1, \ldots, t), n_{i}>0(i=1, \ldots, t) h \geq 0, g \geq 0 \quad$ (not both are zero simultaneously).

$$
\begin{aligned}
& \int_{-1}^{1}(1-x)^{\rho}(1+x)^{\sigma} S_{V}^{U_{1} \ldots U_{t}}\left[y_{1}(1-x)^{m_{1}}(1+x)^{n_{1}}, \ldots, y_{t}(1-x)^{m_{t}}(1+x)^{n_{t}}\right] \\
& \times I_{p_{i}, q_{i}: r}^{m, n}\left[z(1-x)^{g}(1+x)^{h}\left[\begin{array}{l}
{\left[\left(a_{j}, \alpha_{j}\right)_{1, n}\right],\left[\left(a_{j i}, \alpha_{j i}\right)_{n+1, p_{p}}\right]} \\
{\left[\left(b_{j}, \beta_{j}\right)_{1, n}\right],\left[\left(b_{j i}, \beta_{j i}\right)_{n+1, p_{i}}\right]}
\end{array}\right] \mathrm{d} x\right. \\
& =2^{\rho+\sigma+1} \sum_{k_{1} \ldots, k_{t}=0}^{U_{1} k_{1}+\ldots+U_{t} k_{t}}\left(-V_{1}\right)_{U_{1} k_{1}+\ldots+U_{t} k_{t}} A\left(V_{1}, k_{1} ; \ldots ; k_{t}\right) \frac{y_{1}^{k_{1}}}{k_{1} !} \ldots \frac{y_{t}^{k_{t}}}{k_{t} !} 2^{\sum_{i=1}^{t}\left(m_{i}+n_{i}\right) k_{i}} \\
& \times I_{p_{i}+2, \mathrm{q}_{i}+1: r}^{\mathrm{m}, \mathrm{n}+2}\left[\left.z 2^{h+g}\right|_{\left[\left(b_{j}, \beta_{j}\right)_{1, \mathrm{~m}}\right],\left[\left(b_{j i}, \beta_{j i}\right)_{m+1, q_{i}}\right],\left(-\sigma-\rho-\sum_{i=1}^{t}\left(m_{i}+n_{i}\right) k_{i}-1, h+g ; 1\right)} ^{\left(-\sigma \sum_{i=1}^{t} m_{i} k_{i}, h ; 1\right),\left(-\rho-\sum_{i=1}^{t} n_{i} k_{i}, g ; 1\right),\left[\left(a_{j}, \alpha_{j}\right)_{1, \mathrm{n}}\right],\left[\left(a_{j i}, \alpha_{j i}\right)_{n+1, p_{i}}\right]}\right],
\end{aligned}
$$

Provided the conditions stated in results (3.1) are satisfied.

Proof : To establish integral in (3.1), we express I-function occurring in its left -hand side interms of Mellin-Barnes [10] contour integral given by (3.1), the second class of polynomial given by (2.1). Then interchange the order of integration of summations and integration, we arrive the following

$$
\begin{aligned}
& \sum_{k_{1}=0}^{\left[V_{1} / U_{1}\right]} \ldots \sum_{k_{t}=0}^{\left[V_{,}, U_{t}\right]}\left(-V_{1}\right)_{U_{t} k_{1}} \ldots\left(-V_{t}\right)_{U_{t} k_{t}} A\left(V_{1}, k_{1} ; \ldots ; V_{t}, k_{t}\right) \frac{y_{1}^{k_{1}}}{k_{1} !} \ldots \frac{y_{t}^{k_{t}}}{k_{t} !} \\
& \mathrm{x} \frac{1}{2 \pi i} \int_{L} \phi(s) z^{s} \int_{-1}^{1}(1-x)^{\rho+g s+\sum_{i=1}^{t} m_{i} k_{i}}(1+x)^{\sigma+h x+\sum_{i=1}^{t} n_{1} k_{i}} d x d s \\
& =\sum_{k_{1}=0}^{\left[V_{1} / U_{1}\right]} \ldots \sum_{k_{t}=0}^{\left[V_{1},\left\langle U_{t}\right]\right.}\left(-V_{1}\right)_{U_{1} k_{i}} \ldots\left(-V_{t}\right)_{U_{t} k_{t}} A\left(V_{1}, k_{1} ; \ldots ; V_{t}, k_{t}\right) \frac{y_{1}^{k_{1}}}{k_{1} !} \ldots \frac{y_{t}^{k_{t}}}{k_{t} !} \\
& \times \frac{1}{2 \pi i} \int_{L} \phi(s) z^{s} d s 2^{\sigma+h s+\sum_{i=1}^{t} n_{k} k_{i}+\rho+g s+\sum_{i=1}^{t} m_{i} k_{i}+1} \frac{\Gamma\left(\sigma+h s+\sum_{i=1}^{t} n_{i} k_{i}+1\right) \Gamma\left(\rho+g s+\sum_{i=1}^{t} m_{i} k_{i}+1\right)}{\Gamma\left(\sigma+h s+\sum_{i=1}^{t} n_{i} k_{i}+\rho+g s+\sum_{i=1}^{t} m_{i} k_{i}+2\right)}
\end{aligned}
$$

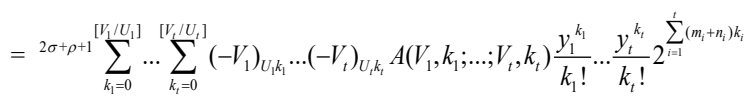$$
\times \frac{1}{2 \pi i} \int_{L} \phi(s) z^{s} \frac{\Gamma\left(\sigma+h s+\sum_{i=1}^{t} n_{i} k_{i}+1\right) \Gamma\left(\rho+g s+\sum_{i=1}^{t} m_{i} k_{i}+1\right)}{\Gamma\left(\sigma+h s+\sum_{i=1}^{t} n_{i} k_{i}+\rho+g s+\sum_{i=1}^{t} m_{i} k_{i}+2\right)}
$$

$\left(z 2^{h+g}\right)^{s} d s$

Now we evaluate the above integral with help of integral (2.2). Interpreting the resulting contour integral of $\mathrm{H}$-function we can easily arrive at desired result (3.1).

To establish integral in (3.2) can be easily established on the same lines similar to the proof of (3.1).

\section{Special Cases of (3.1) and (3.2)}

Take A $\left(\mathrm{V}_{1}, \mathrm{k}_{1} ; \ldots ; \mathrm{V}_{\mathrm{t}}, \mathrm{k}_{\mathrm{t}}\right)=\mathrm{A}_{1}\left(\mathrm{~V}_{1}, \mathrm{k}_{1}\right) \ldots \mathrm{A}_{\mathrm{t}}\left(\mathrm{V}_{\mathrm{t}}, \mathrm{k}_{\mathrm{t}}\right)$ in (3.1) the multivariable polynomial $S_{V_{1} \ldots V_{t}}^{U_{1} \ldots U_{t}}\left(x_{1}, \ldots, x_{t}\right)$ reduced to the product of wellknown general class of polynomials $S_{v}^{U}(x)$ and the result (3.1) reduced to following form

$$
\begin{aligned}
& \int_{-1}^{1}(1-x)^{\rho}(1+x)^{\sigma} \prod_{i=1}^{t} S_{V_{i}}^{U_{i}}\left[y_{i}(1-x)^{m}(1+x)^{n}\right] \\
& \times I_{p_{i}, q_{i}: r}^{m, n}\left[z(1-x)^{g}(1+x)^{h}\left[\begin{array}{l}
{\left[\left(a_{j}, \alpha_{j}\right)_{1, n}\right],\left[\left(a_{j i}, \alpha_{j i}\right)_{n+1, p_{i}}\right]} \\
{\left[\left(b_{j}, \beta_{j}\right)_{1, n}\right],\left[\left(b_{j i}, \beta_{j i}\right)_{n+1, \mathrm{q}_{i}}\right]}
\end{array}\right] \mathrm{dx}\right. \\
& \sum_{2^{\sigma}=0}^{\sigma+\rho+1}\left[\sum_{\left.k_{1} / U_{1}\right]}^{\left[V_{t} / U_{t}\right]}\left(-V_{1}\right)_{U_{1} k_{i}} \ldots\left(-V_{t}\right)_{U_{t} k_{t}} A\left(V_{1}, k_{1} \ldots, V_{t} k_{t}\right) \frac{y_{1}^{k_{1}}}{k_{1} !} \ldots \frac{y_{t}^{k_{t}}}{k_{t} !} 2^{\sum_{i=1}^{t}\left(m_{i}+n_{i}\right) k_{i}}\right. \\
& \times I_{p_{i}+2, \mathrm{q}_{i}+1: r}^{\mathrm{m}, \mathrm{n}+2}\left[\left.z 2^{h+g}\right|_{\left[\left(b_{j}, \beta_{j}\right)_{1, \mathrm{~m}}\right],\left[\left(b_{j i}, \beta_{j i}\right)_{m+1, q_{i}}\right],\left(-\sigma-\rho-\sum_{i=1}^{t}\left(m_{i}+n_{i}\right) k_{i}-1, h+g ; 1\right)} ^{\left(-\sigma-\sum_{i=1}^{t} m_{i} k_{i}, h, 1\right),\left(-\rho-\sum_{i=1}^{t} n_{i} k_{i}, g ; 1\right)\left[\left(a_{j}, \alpha_{j}\right)_{1, \mathrm{n}}\right],\left[\left(a_{j i}, \alpha_{j i}\right)_{n+1, \mathrm{p}_{i}}\right]}\right]
\end{aligned}
$$

\section{(a)Substituting $r=1$ in (3.1), we obtain :}

$$
\begin{aligned}
& \int_{-1}^{1}(1-x)^{\rho}(1+x)^{\sigma} \mathrm{S}_{V_{1}, \ldots, V_{t}}^{U_{1}, \ldots, U_{t}}\left[\mathrm{y}_{1}(1-x)^{m_{1}}(1+x)^{n_{1}}, \ldots, \mathrm{y}_{t}(1-x)^{m_{t}}(1+x)^{n_{t}}\right] \\
\times & \mathrm{H}_{p, q}^{m, n}\left[z(1-x)^{g}(1+x)^{h} \mid \begin{array}{l}
\left(a_{j}, \alpha_{j}\right)_{1, p} \\
\left(b_{j}, \beta_{j}\right)_{1, q}
\end{array}\right] \mathrm{d} x \\
= & 2^{\rho+\sigma+1} \sum_{K_{1}=0}^{\left[V_{1}, U_{1}\right]} \ldots \ldots . \sum_{K_{t}=0}^{\left[V_{,}\left(U_{1}\right]\right.} \frac{\left(-V_{1}\right)_{U_{U}, k_{1}}}{k_{1} !} \ldots \frac{\left(-V_{t}\right)_{U_{U}, K_{t}}}{k_{t} !} \mathrm{A}\left[\mathrm{V}_{1}, k_{1} ; \ldots ; \mathrm{V}_{t}, k_{t}\right] \frac{y_{1}^{k_{1}}}{k_{1} !} \ldots \frac{y_{t}^{k_{t}}}{k_{t} !} \sum_{i=1}^{t}\left(m_{i}+n_{i}\right) \mathrm{k}_{i} \\
\times & \mathrm{H}_{p, q}^{m, n}\left[z 2^{h+g} \mid \begin{array}{l}
\left(-\sigma-\sum_{i=1}^{t} m_{i} k_{i}, h ; 1\right),\left(-\rho-\sum_{i=1}^{t} n_{i} k_{i}, g ; 1\right)\left(a_{j}, \alpha_{j}\right)_{1, \mathrm{p}} \\
\left(b_{j}, \beta_{j}\right)_{1, \mathrm{q}}\left(-\sigma-\rho-\sum_{i=1}^{t}\left(m_{i}+n_{i}\right) k_{i}-1, h+g ; 1\right)
\end{array}\right]
\end{aligned}
$$

\section{(b)Substituting $\alpha_{j}=\beta_{j}=1$ in (4.2) we obtain}

$$
\begin{aligned}
& \int_{-1}^{1}(1-x)^{\rho}(1+x)^{\sigma} S_{V_{1} \ldots V_{t}}^{U_{1} \ldots U_{t}}\left[y_{1}(1-x)^{m_{1}}(1+x)^{n_{1}} \ldots y_{t}(1-x)^{m_{t}}(1+x)^{n_{t}}\right] \\
& \times G_{\mathrm{p}, \mathrm{q}}^{\mathrm{m}, \mathrm{n}}\left[\left.z(1-x)^{g}(1+x)^{h}\right|_{\left(b_{j}\right)_{1, \mathrm{q}}} ^{\left(a_{j}\right)_{1, \mathrm{p}}}\right] d x \\
& \sum_{k_{1}=0} \ldots \sum_{k_{t}=0}^{2^{\sigma+\rho+1}}\left(-V_{1}\right)_{U_{1} k_{1}, \ldots\left(U_{1}\right]} \ldots\left(-V_{t}\right)_{U_{t} k_{t}} A\left(V_{1}, k_{1} \ldots, V_{t}, k_{t}\right) \frac{\left.y_{1}^{k_{1}} / U_{t}\right]}{k_{1} !} \ldots \frac{y_{t}^{k_{t}}}{k_{t} !} 2^{\sum_{i=1}^{t}\left(m_{i}+n_{i}\right) k_{i}} \\
& \times G_{p+2, \mathrm{q}+1}^{\mathrm{m}, \mathrm{n}+2}\left[\left.z 2^{h+g}\right|_{\left(b_{j}\right)_{1, \mathrm{q}},\left(-\sigma-\rho-\sum_{i=1}^{t}\left(m_{i}+n_{i}\right) k_{i}-1, h+g ; 1\right)} ^{\left.\left(-\sigma-\sum_{i=1}^{t} m_{i} k_{i}, h, 1\right),\left(-\rho-\sum_{i=1}^{t} n_{i} k_{i}, g ; 1\right)\left(a_{j}\right)_{1, \mathrm{p}}\right] .}\right.
\end{aligned}
$$

(a)Substituting $r=1$ in (3.2), we obtain :

$$
\begin{aligned}
& \int_{-1}^{1}(1-x)^{p}(1+x)^{q} S_{V}^{U_{1} \ldots U_{t}}\left[y_{1}(1-x)^{m}(1+x)^{n}, \ldots, y_{t}(1-x)^{m}(1+x)^{n}\right] \\
& \times \mathrm{H}_{p, q}^{m, n}\left[z(1-x)^{g}(1+x)^{h} \mid \begin{array}{l}
\left(a_{j}, \alpha_{j}\right)_{1, \mathrm{p}} \\
\left(b_{j}, \beta_{j}\right)_{1, q}
\end{array}\right] \mathrm{d} x
\end{aligned}
$$




$$
\begin{aligned}
& =2^{\rho+\sigma+1} \sum_{k_{1} \ldots, k_{t}=0}^{U_{1} k_{1}+\ldots+U_{t} k_{t}}\left(-V_{1}\right)_{U_{1} k_{1}+\ldots+U t k t} A\left(V_{1}, k_{1} ; \ldots, k_{t}\right) \frac{y_{1}^{k_{1}}}{k_{1} !} \ldots \frac{y_{t}^{k_{t}}}{k_{t} !} 2^{\sum_{i=1}^{t}\left(m_{i}+n_{i}\right) k_{i}} \\
& \times H_{p+2, \mathrm{q}+1}^{\mathrm{m}, \mathrm{n}+2}\left[\left.z 2^{h+g}\right|_{\left(b_{j}, \beta_{j}\right)_{1, \mathrm{q}},\left(-\sigma-\rho-\sum_{i=1}^{t}\left(m_{i}+n_{i}\right) k_{i}-1, h+g ; 1\right)} ^{\left(-\sigma-m_{i} k_{i}, h, 1\right),\left(-\rho-\sum_{i=1}^{t} n_{i} k_{i}, g ; 1\right),\left(a_{j}, \alpha_{j}\right)_{1, \mathrm{p}}}\right]
\end{aligned}
$$

(b)Substituting $\alpha_{j}=\beta_{j}=1$ in (4.4), we obtain

$$
\begin{aligned}
& \int_{-1}^{1}(1-x)^{\rho}(1+x)^{\sigma} S_{V}^{U_{1} \ldots U_{t}}\left[y_{1}(1-x)^{m}(1+x)^{n}, \ldots, y_{t}(1-x)^{m}(1+x)^{n}\right] \\
& \times \mathrm{G}_{p, q}^{m, n}\left[z(1-x)^{g}(1+x)^{h}\left[\begin{array}{l}
\left(a_{j}\right)_{1, \mathrm{p}} \\
\left(b_{j}\right)
\end{array}\right] \mathrm{d} x\right. \\
& =2^{\rho+\sigma+1} \sum_{k_{1} \ldots, k_{t}=0}^{U_{1} k_{1}+\ldots+U_{t} k_{t}}\left(-V_{1}\right)_{U_{1} k_{1}+\ldots+U_{t} k_{t}} A\left(V_{1}, k_{1} ; \ldots, k_{t}\right) \frac{y_{1}^{k_{1}}}{k_{1} !} \ldots \frac{y_{t}^{k_{t}}}{k_{t} !} 2^{\sum_{i=1}^{t}\left(m_{i}+n_{i}\right) k_{i}} \\
& \times G_{p+2, \mathrm{q}+1}\left[\left.z 2^{h+g}\right|_{\left(b_{j}\right)_{1, \mathrm{q}},\left(-\sigma-\rho-\sum_{i=1}^{t}\left(m_{i}+n_{i}\right) k_{i}-1, h+g ; 1\right)} ^{\left.\left(-\sigma-\sum_{i=1}^{t} m_{i} k_{i}, h, 1\right),\left(-\rho-\sum_{i=1}^{t} n_{i} k_{i}, g ; 1\right),\left(a_{j}\right)_{1, \mathrm{p}}\right]}\right.
\end{aligned}
$$

\section{References}

1. Saxena S (1982) Formal solution of certain new pair of dual integral equations involving H-function. Proc Nat Acad Sci India 52, A. III, 366-375.

2. Gradshteyin IS, Ryzhik IM (2001) Table of Integrals, series and products, 6/e. Academic press, New Delhi.

3. Sharma RP (2006) On finite integrals involving Jacobi polynomials and the $\mathrm{H}$-function. Kyungpook. Math J 46: 307-313.

4. Srivastava HM, Garg M (1987) Some integrals involving a general class of polynomials and the multivariable H-function. Rev Roamaine Phys 32: 685-692.

5. Satyanarayana B, Pragathi Kumar Y (2011) Some finite integrals involving multivariable polynomials, $\mathrm{H}$-function of one variable and $\mathrm{H}$-function of ' $\mathrm{r}$ ' variables. African Journal of Mathematics and Computer Science Research 4: 281-285.

6. Agarwal P (2012) On a unified integral involving the product of Srivastava's polynomials and generalized Mellin-Barnes type of contour integral. Advances in Mechanical Engineering and its Applications (AMEA) 158: 2.

7. Bhattar B (2014) Integral formulae's involving two $\bar{H}$ - function and multivariable's general class of polynomiyals. ISCA Bushma.

8. Srivastava A (2010) The integration of certain products pertaining to the H-function with general polynomials. Ganita Sandesh 31/32: 51-58.

9. Srivatsava HM, Singh NP (1983) The integration of certain products of the multivariable $\mathrm{H}$-function with a general class of polynomials. Rend Circ Mat Palermo 32.

10. Agarwal P, Chand M (2012) New theorems involving the generalized Mellin-Barnes type of contour integrals and general class of polynomials. GJSFRM 12

Copyright: (C2016 Mishra J. This is an open-access article distributed under the terms of the Creative Commons Attribution License, which permits unrestricted use, distribution, and reproduction in any medium, provided the original author and source are credited. 